ARTICLE

\title{
Structural basis for activation of the growth hormone-releasing hormone receptor
}

Fulai Zhou,2,11, Huibing Zhang 3,11, Zhaotong Cong4,11, Li-Hua Zhao 1,11, Qingtong Zhou (1) 5,11, Chunyou Mao ${ }^{3}$, Xi Cheng (1) 6,7, Dan-Dan Shen ${ }^{3}$, Xiaoqing Cai ${ }^{1,2}$, Cheng Ma ${ }^{3}$, Yuzhe Wang ${ }^{1,2,8}$, Antao Dai ${ }^{1,2}$, Yan Zhou ${ }^{1,2}$, Wen Sun 1,2,8, Fenghui Zhao ${ }^{4}$, Suwen Zhao (1) 5,9, Hualiang Jiang (1) 6,7,8,9, Yi Jiang (1) ${ }^{1}$, Dehua Yang (1) 1,2,8凶, H. Eric Xu (D) ${ }^{1,8 凶}$, Yan Zhang (iD ${ }^{3 凶}$ \& Ming-Wei Wang (1) $1,2,4,8,9,10 \bowtie$

Growth hormone-releasing hormone $(\mathrm{GHRH})$ regulates the secretion of growth hormone that virtually controls metabolism and growth of every tissue through its binding to the cognate receptor (GHRHR). Malfunction in GHRHR signaling is associated with abnormal growth, making GHRHR an attractive therapeutic target against dwarfism (e.g., isolated growth hormone deficiency, IGHD), gigantism, lipodystrophy and certain cancers. Here, we report the cryo-electron microscopy (cryo-EM) structure of the human GHRHR bound to its endogenous ligand and the stimulatory $G$ protein at 2.6 $\AA$. This high-resolution structure reveals a characteristic hormone recognition pattern of GHRH by GHRHR, where the $\alpha$-helical GHRH forms an extensive and continuous network of interactions involving all the extracellular loops (ECLs), all the transmembrane (TM) helices except TM4, and the extracellular domain (ECD) of GHRHR, especially the N-terminus of GHRH that engages a broad set of specific interactions with the receptor. Mutagenesis and molecular dynamics (MD) simulations uncover detailed mechanisms by which IGHD-causing mutations lead to the impairment of GHRHR function. Our findings provide insights into the molecular basis of peptide recognition and receptor activation, thereby facilitating the development of structure-based drug discovery and precision medicine.

\footnotetext{
${ }^{1}$ The CAS Key Laboratory of Receptor Research, Shanghai Institute of Materia Medica, Chinese Academy of Sciences, 201203 Shanghai, China. ${ }^{2}$ The National Center for Drug Screening, Shanghai Institute of Materia Medica, Chinese Academy of Sciences, 201203 Shanghai, China. ${ }^{3}$ Department of Biophysics, and Department of Pathology of Sir Run Run Shaw Hospital, Zhejiang University School of Medicine, 310058 Hangzhou, China. ${ }^{4}$ School of Pharmacy, Fudan University, 201203 Shanghai, China. ${ }^{5}$ iHuman Institute, ShanghaiTech University, 201210 Shanghai, China. ${ }^{6}$ State Key Laboratory of Drug Research, Shanghai Institute of Materia Medica, Chinese Academy of Sciences, 201203 Shanghai, China. ${ }^{7}$ Drug Discovery and Design Center, Shanghai Institute of Materia Medica, Chinese Academy of Sciences, 201203 Shanghai, China. ${ }^{8}$ University of Chinese Academy of Sciences, 100049 Beijing, China. ${ }^{9}$ School of Life Science and Technology, ShanghaiTech University, 201210 Shanghai, China. ${ }^{10}$ School of Basic Medical Sciences, Fudan University, 200032

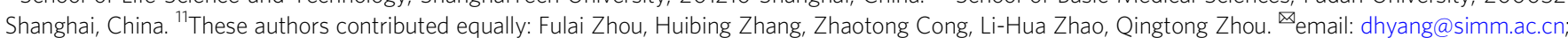
eric.xu@simm.ac.cn; zhang_yan@zju.edu.cn; mwwang@simm.ac.cn
} 
C lass B G-protein-coupled receptors (GPCRs) are key players in hormonal homeostasis and important drug targets for endocrinal and neuronal disorders. Growth hormonereleasing hormone receptor (GHRHR), a prototypical class B GPCR, is expressed by somatotropic cells of the pituitary gland. Activation of GHRHR by GHRH, a 44-amino acid peptide released by the hypothalamus ${ }^{1,2}$, results in the secretion and production of growth hormone $(\mathrm{GH})$ through cyclic adenosine monophosphate (cAMP)-dependent pathways ${ }^{3}$. Numerous studies demonstrated that GHRH exerts a variety of bioactivities due to its wide distribution and autocrine/paracrine mechanisms ${ }^{4,5}$. Therefore, GHRH and its analogs, including tesamorelin, MR-409, JI-38, and MIA-690, have been developed as potential therapeutic agents to treat diabetes, cancers, and cardiovascular diseases ${ }^{5-9}$.

Like other class B GPCRs, GHRHR consists of an extracellular domain (ECD) and a seven-transmembrane helix domain $(7-T M D)^{10,11}$. Recently published cryo-electron microscopy (cryo-EM) structures of class B GPCRs bound to a $\mathrm{G}_{\mathrm{s}}$ heterotrimer protein include parathyroid hormone receptor 1 (PTH1R), glucagon-like peptide-1 receptor (GLP-1R), calcitonin receptor, calcitonin gene-related peptide receptor (CGRPR), two subtypes of corticotrophin-releasing factor (CRF1R and CRF2R), and adrenomedullin receptors (AM1R and AM2R), as well as pituitary adenylate cyclase-activating polypeptide (PACAP) type I receptor $(\mathrm{PAC1R})$ and vasoactive intestinal polypeptide receptor (VIP1R), revealing a common mode of ligand-induced receptor activation $^{10-19}$. The C-terminal a helix of peptide ligand recognizes and binds to the ECD, thereby allowing its $\mathrm{N}$-terminus to interact with the extracellular TM core. This is followed by a major conformational change that involves a large kink at the TM6 to open the intracellular face for G protein coupling ${ }^{15}$. However, ligand-binding specificity and roles of ECD in receptor activation vary widely among class B GPCRs due to diverse amino acid sequences of both peptidic ligands and receptors ${ }^{15,20}$.

Here, we employed the single-particle cryo-EM approach to determine the near-atomic resolution structure of the human GHRHR bound to GHRH in complex with a heterotrimeric $G_{s}$ protein. Together with functional studies and molecular dynamics (MD) simulations, our results provide key insights into the structural basis of ligand recognition, receptor activation, and isolated growth hormone deficiency (IGHD) causing mechanism related to GHRHR, thereby offering a template for rational design of drugs against this receptor.

\section{Results}

Structure determination of the GHRH-GHRHR-G complex. We developed a NanoBiT tethering strategy to stabilize the assembly of a GHRH-GHRHR-G $\mathrm{G}_{\mathrm{s}}$ complex for cryo-EM studies, overcoming the lack of stability of the above complex (Supplementary Fig. 1 and Supplementary Table 1), as it has been used for the VIP1R-G complex $^{19}$. Using this approach, we were able to obtain a GHRH-GHRHR-G $\mathrm{G}_{\mathrm{s}}$ complex with improved homogeneity and stability (Supplementary Fig. 2). The GHRH-GHRHR-G complex was vitrified and cryo-EM images were collected under a Titan Krios microscope equipped with $\mathrm{K} 2$ summit direct detector. The structure of GHRH-GHRHR- $\mathrm{G}_{\mathrm{s}}$ complex was determined from 307,018 particles to an overall resolution of $2.6 \AA$ (Supplementary Figs. 3 and 4). The resulting model contains 28 residues of GHRH (residues 1-28), Gaßy subunits except the $\alpha$-helical domain (AHD) of $\mathrm{Ga}_{\mathrm{s}}$, and GHRHR residues from 119 to 394. Besides, the ECD region of GHRHR was not resolvable with this limited dataset, perhaps reflecting its highly dynamic and conformationally flexible property when bound to GHRH. We rigid-body fitted the GHRHR ECD (residues 25-118) crystal structure (PDB accession: 2XDG) to the low-pass filtered map (Fig. 1b). The majority of amino acid side a

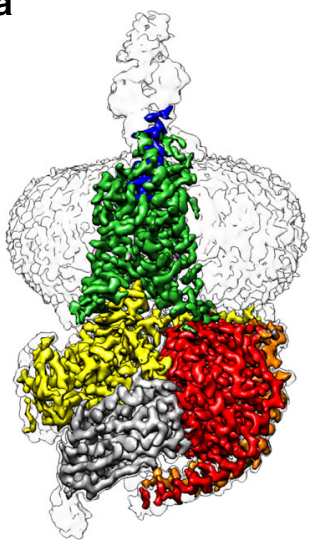

C

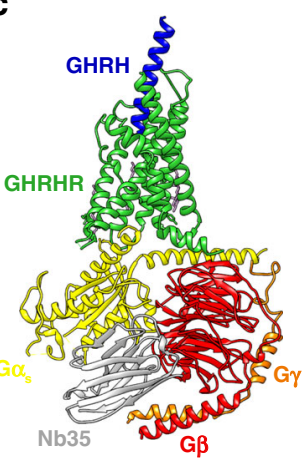

b

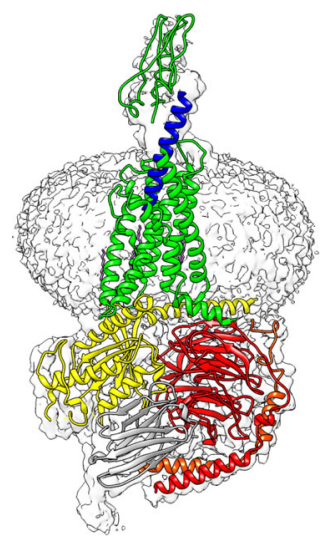

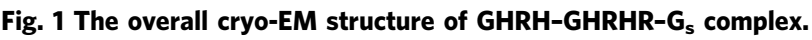
a Cryo-EM density map that illustrates the GHRH-GHRHR-G ${ }_{\mathrm{s}}$ complex and the disc-shaped micelle. The unsharpened cryo-EM density map at the 0.005 threshold shown as light gray surface indicates a micelle diameter of $10 \mathrm{~nm}$. The colored cryo-EM density map is shown at 0.028 threshold. b GHRH-GHRHR-G complex model and GHRHR ECD crystal structure model docked into the cryo-EM map. c Cartoon representation of the

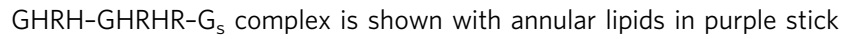
representation. Lime green, GHRHR; blue, $\mathrm{GHRH}$; yellow, $\mathrm{G}_{\mathrm{s}}$ Ras-like domain; red, G $\beta$; orange, G $\gamma$; gray, Nb35; plum, lipid, and cholesterol.

chains were well resolved in the final model (Supplementary Fig. 5), which was refined against the EM density map (Fig. 1a) with excellent geometry (Supplementary Table 2). Owing to the highresolution map, we identified one water molecule in the orthosteric binding site, and two water molecules in the $G$ protein engaging pocket. Akin to cryo-EM structure of PTH1R-G $\mathrm{G}_{\mathrm{s}}$ complex, the TMD of GHRHR is surrounded by annular detergent micelle with a diameter of $10 \mathrm{~nm}$, mimicking the natural phospholipid bilayer. Within the micelle, one bound cholesterol and two lipids are also clearly visible in the cryo-EM map.

Molecular recognition of GHRH by GHRHR. In the complex structure, GHRH adopts an $\alpha$-helical configuration when engaged with GHRHR. Compared to GLP-1 (ref. ${ }^{10}$ ) and long-acting parathyroid hormone analog (LA-PTH) ${ }^{15}$, GHRH binds to the GHRHR TMD through a more extensive and continuous network of interactions involving all the extracellular loops (ECLs), all the TM helices except TM4, and the linker connecting ECD and TMD. The N-terminus of GHRH inserts deeply into the TMD core and engages an extensive set of receptor-specific interactions (Fig. 2a-c and Supplementary Table 3 ). Interestingly, $\mathrm{Tyr}^{1 \mathrm{P}}$ locates in the equivalent position of the second residue in other class B GPCR peptidic agonists, such as exendin-P5 (ExP5) and LA-PTH, where 
a

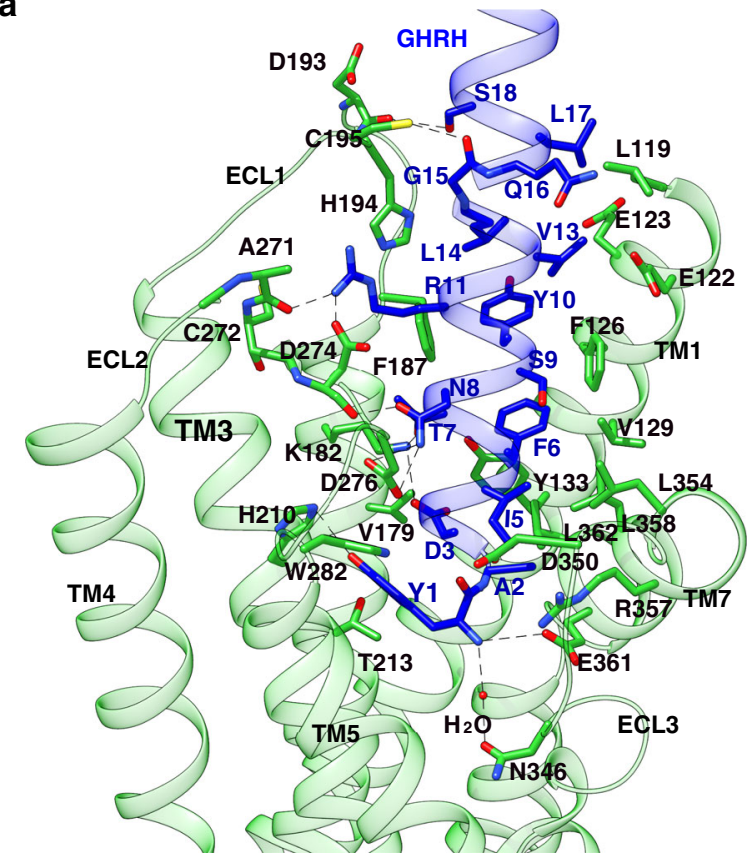

b

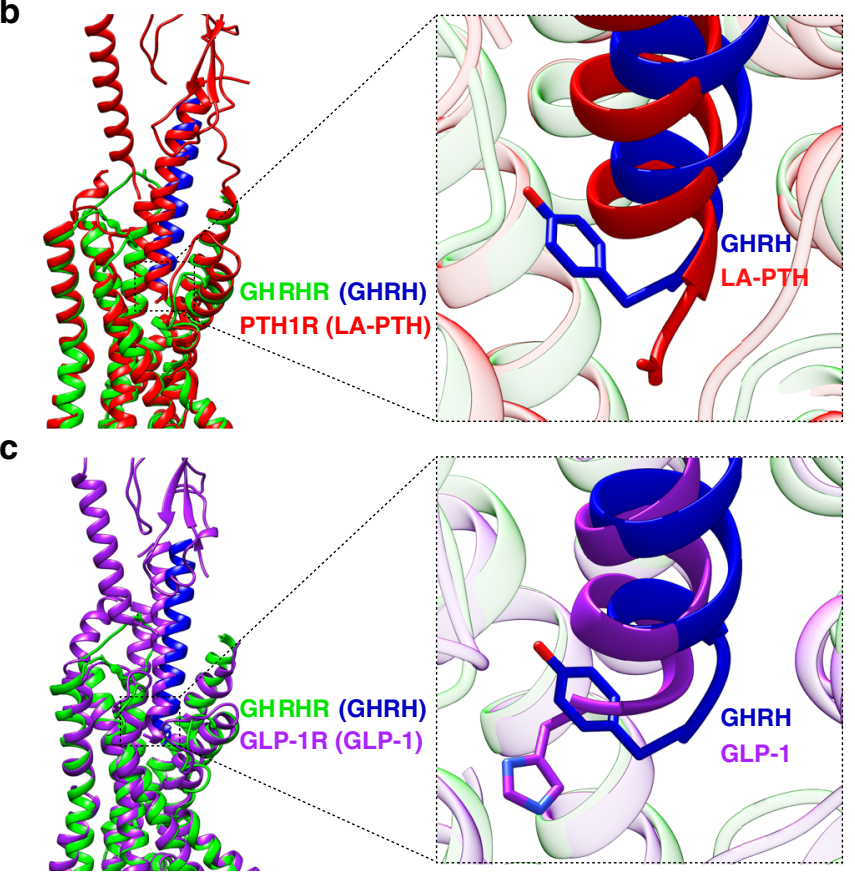

d

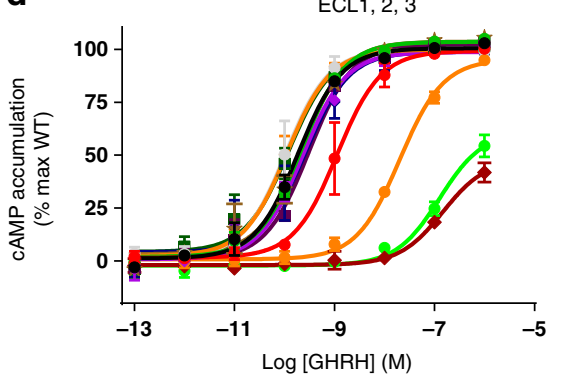

e
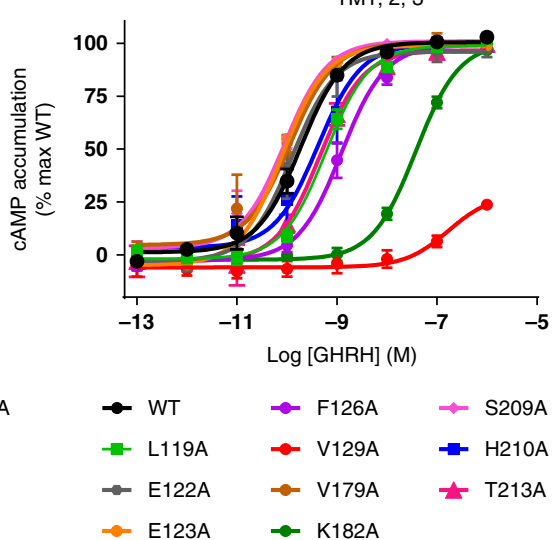

f

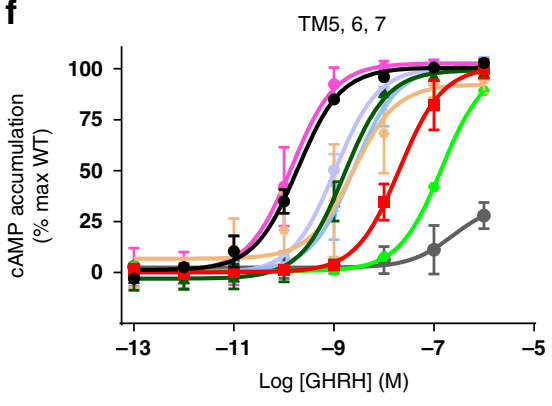

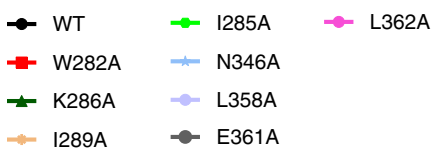

Fig. 2 Molecular recognition of GHRH by GHRHR and comparison of that with LA-PTH-PTH1R and GLP-1-GLP-1R. a Detailed interaction between GHRH and the TMD pocket of GHRHR with hydrogen bonds shown as dotted lines. b, c Pairwise comparison of GHRH bound to GHRHR with GLP-1 and LA-PTH in complex with their corresponding receptors, showing the relative positions of peptide ligands. Lime green, GHRHR; blue, GHRH; red, PTH1R and LA-PTH; purple, GLP-1R and GLP-1. d-f The effects of mutation in the ligand-binding pocket on CAMP accumulation. cAMP levels were measured in wild-type (WT) receptor and alanine mutants in ECL1, 2, and 3 (d), TM1, 2, and 3 (e), and TM5, 6, and 7 (f). cAMP signals were normalized to the maximum response of WT and concentration-response curves were analyzed using a three-parameter logistic equation. Data shown are means \pm S.E.M. of at least three independent experiments $(n=3-5)$, conducted in triplicate. Source data are provided as a Source data file.

their first residues of the side chain have different orientations (Fig. 2b). The hydroxyl group of $\mathrm{Tyr}^{1 \mathrm{P}}$ forms hydrogen bonds with $\mathrm{H} 210^{3.37 \mathrm{~b}}$ (class B GPCR numbering in superscript) ${ }^{21}$ and van der Waals interactions with $\mathrm{T} 213^{3.40 \mathrm{~b}}$ and $\mathrm{W} 282^{5.36 \mathrm{~b}}$, whereas the main chain $\mathrm{NH}$ of $\mathrm{Tyr}^{1 \mathrm{P}}$ forms hydrogen bond with R357 $7.38 \mathrm{~b}$ of TM7 and contacts a water molecule that connects with N346 $6.57 \mathrm{~b}$ of TM6-ECL3 hinge, possibly stabilizing the ECL3 in an active state. This observation is consistent with our observation that impairing these contacts dramatically decreased the potency of GHRH in stimulating cAMP accumulation (Fig. $2 \mathrm{~d}-\mathrm{f}$ and Supplementary Table 4). The most conserved Asp/Glu at position 3 across peptide hormones of the glucagon receptor (GCGR) subfamily, $\mathrm{Asp}^{3 \mathrm{P}}$ in the case of GHRH, forms salt bridges with $\mathrm{K} 182^{2.67 \mathrm{~b}}$, which is further strengthened by a polar network composed of $\mathrm{Thr}^{7 \mathrm{P}}$, Y133 $1.43 \mathrm{~b}$, and D183 $2.68 \mathrm{~b}$. From evolutional biology perspective, both $\mathrm{Asp}^{3 \mathrm{P}}$ and $\mathrm{K} 182^{2.67 \mathrm{~b}}$ are fully conserved for GHRH and GHRHR from dozens of species (Supplementary Fig. $6 \mathrm{a}-\mathrm{b})$. Indeed, $\mathrm{D}^{3 \mathrm{P}} \mathrm{A}$ and $\mathrm{K} 182^{2.67 \mathrm{~b}} \mathrm{~A}$ diminished the potency of GHRH by 4- and 200-fold (Fig. 2e and Supplementary Fig. 6c), respectively. The combined structural, pharmacological, and evolutional investigations point to a crucial role of $\mathrm{Asp}^{3 \mathrm{P}}$ in peptide binding and receptor activation. $\mathrm{Phe}^{6 \mathrm{P}}$ is another conserved residue and contributes extensive contacts with surrounding residues of GHRHR, including Pi-Pi stacking with F126 $1.36 \mathrm{~b}$ and Y133 $1.43 \mathrm{~b}$, and hydrophobic contacts with V1291.39b and L362 ${ }^{7.43 b}$, where these four residues are either fully conserved or physiochemically similar among GHRHRs of different species. By forming salt bridges with D274 ${ }^{5.52 \mathrm{~b}}$ and hydrogen bonds with backbone atoms of $\mathrm{H} 194^{\mathrm{ECL} 1}$ and A271 ${ }^{\mathrm{ECL} 2}$, fully conserved $\mathrm{Arg}^{11 \mathrm{P}}$ greatly stabilizes the ECL1-ECL2 interface relative to the conserved TM3ECL2 disulfide bond (Cys ${ }^{3.29 b}-\mathrm{Cys}^{\mathrm{ECL} 2}$ ) of class B GPCRs. This interface is further stabilized by close contacts between ECL1 and 
GHRH (T-shape Pi stacking between $\mathrm{Tyr}^{10 \mathrm{P}}$ and F187 ${ }^{\mathrm{ECL} 1}$, and hydrogen bonds between $\operatorname{Ser}^{18 \mathrm{P}}$ and D193 ${ }^{\mathrm{ECL} 1}$ ), as well as between ECL2 and GHRH (hydrogen bonds between Asn ${ }^{8 \mathrm{P}}$ and D274). For residues like $\mathrm{Ala}^{2 \mathrm{P}}$ and $\mathrm{Ile}^{5 \mathrm{P}}$, they contribute hydrophobic interactions with $\mathrm{L} 362^{7.43 \mathrm{~b}}$ and D350 $\mathrm{ECL3}$, which are conserved among different species. The remaining residues, including $\mathrm{Ala}^{4 \mathrm{P}}, \mathrm{Ser}^{9 \mathrm{P}}$, and $\mathrm{Gly}^{15 \mathrm{P}}$, are variable and receptive to substitution by Ala, Gly, Cys, or Ser, indicating their role of structural complementarily. Amino acids of the peptide after 20 may have rich interactions with the ECD as indicated by the model, in which the ECD was docked in the EM map as a rigid body (Fig. 1b). Indeed, cAMP signaling was nearly abolished in HEK 293 T cells expressing a truncated ECD construct, i.e., GHRHR(119-423), suggesting an essential role of ECD in GHRH recognition and receptor activation (Supplementary Fig. 7a). In our $1 \mu$ s MD simulation, the ECD of GHRHR was able to twist around the GHRH helix, while the TMD core is quite stable in a single dominant conformational state as seen previously with PTH1R ${ }^{15}$. Specifically, massive hydrophobic contacts between GHRH (R20, L22, L23, and M27) and GHRHR ECD (L34, L62, F82, Y108, P109, and L118), as well as several hydrogen bonds (Q24 of GHRH and C112 of GHRHR), were observed, which may stabilize the binding of GHRH (Supplementary Fig. $7 b$ ). These results are consistent with the findings on the same receptor reported in the literature ${ }^{22,23}$. In fact, such a dynamic and flexible feature of ECD could also be found among other class B GPCRs, such as PTH1R 15 , GLP-1R ${ }^{10}$, and VIP1R ${ }^{19}$, where the conversion from a closed conformation in the apo-state to an extended open conformation is required for activation (Supplementary Fig. 7c).

Unlike the helical extensions found in ECL1 of GCGR, GLP1R, CRF1R, CRF2R, AM1R, and AM2R, and the unstructured ECL1 of PTH1R in active state, the ECL1 of GHRHR stretches around GHRH to form broad interactions. Consistently, disruption of GHRH-ECL interaction by $\mathrm{F} 187^{\mathrm{ECL} 1} \mathrm{~A}$ and $\mathrm{C} 195^{\mathrm{ECL1}} \mathrm{A}$ reduced GHRH potency by $\sim 5-$ and 100 -fold, respectively (Fig. 2d). Coupled with the conformational change from $\beta$ hairpin to $\alpha$-helix in ECL1 of GCGR upon activation ${ }^{24}$, and the limited impact on $\mathrm{G}_{\mathrm{s}}$-mediated cAMP signaling by alanine mutation on ECL1 of GLP-1R ${ }^{25}$, these results demonstrate the dynamic nature and diversified roles of ECL1 in class B GPCRs.

Active structure of GHRHR. The overall arrangement of the GHRH-GHRHR-G $\mathrm{G}_{\mathrm{s}}$ complex is highly similar to GLP-1-GLP$1 R-G_{s}$, glucagon-GCGR-G $G_{s}$, and LA-PTH-PTH1R-G complexes $^{10,14,15}$. Superimposition of TMs shows that active GHRHR, GLP-1R, and PTH1R share similar folds with respect to the global conformation of the 7-TM bundle, as well as a similar organization of the extracellular end of TM6 and TM7 to accommodate peptide ligand. Except ICL3, other loop regions of the receptor are visible due to the high-quality cryo-EM density map, although these loops are relatively dynamic compared to the TMD bundle (Fig. 1c and Supplementary Fig. 5).

Comparison of the GHRHR complex with inactive class B GPCR structures ${ }^{26,27}$, such as GLP-1R and GCGR, suggests that the most obvious conformational transformation is located at TM6 (Fig. 3a-c), where there is a large outward movement at the cytoplasmic face in the activated GHRHR structure upon coupling to $G_{s}$. The TM6 outward displacement is correlated with the kink at the conserved Pro ${ }^{6.47 b}-\mathrm{X}-\mathrm{X}-\mathrm{Gly}^{6.50 \mathrm{~b}}$ motif $^{10,13,16,28}$.

Diversified peptide binding modes. Different from the ligandbinding pockets for small molecules in class A GPCRs, the peptidebinding pockets in class B GPCRs are larger and more extended, involving ECD, ECLs, and TMs. As shown in Fig. 3d, e, class B GPCR agonists adopt diversified conformations and orientations especially in the $\mathrm{N}$ - and C-terminals. Contrary to the single continuous helix observed in GHRH, GLP-1, glucagon, ExP5, PACAP38, and LA-PTH, the N-termini of AM, AM2, CGRP, CRF, and UCN1 looping back between TM5 and TM6, while AM, AM2, and CGRP also have a large kink at the C-terminal portion. Comparison of peptide recognition modes in the GCGR subfamily reveals that binding specificity mainly resides in three segments: $\mathrm{N}$ terminus (first three residues in GHRH), middle region (6th to 11th residues), and C-terminal portion (Fig. 3d, e and Supplementary Fig. 8).

The orientation of the residues at $\mathrm{N}$-terminus are different: the side chain of $\mathrm{Tyr}^{1 \mathrm{P}}$ in GHRH and $\mathrm{His}^{1 \mathrm{P}}$ in glucagon directly face TM3 forming hydrogen bonds with $\mathrm{H} 210^{3.37 \mathrm{~b}}$ and $\mathrm{I} 235^{3.40 \mathrm{~b}}$; the side chain of His $^{7 P}$ in GLP-1 directly faces TM5 and has cation-Pi interaction from upward R299 ${ }^{\mathrm{ECL} 2}$; and the first two residues of LA-PTH $\left(\mathrm{Ala}^{1 \mathrm{P}} \mathrm{Val}^{2 \mathrm{P}}\right)$ and $\operatorname{ExP} 5\left(\mathrm{Glu}^{1 \mathrm{P}} \mathrm{Leu}^{2 \mathrm{P}}\right)$ insert into the cleft between TM5 and TM6. Meanwhile, the orientation of highly conserved Glu/Asp ${ }^{3 \mathrm{P}}$ is significantly different (Supplementary Fig. 8d, e). These observations suggest the complexity of signal initiation.

The middle region of peptides has rich contacts with ECL1 and structurally flexible ECL2 in a peptide-dependent manner (Fig. 3e and Supplementary Fig. 8f). Resulted from longer a-helical extensions of TM2, the elevated ECL1 of GLP-1R and GCGR contribute additional contacts with the peptide C-terminal region. For receptors whose residues at 45.52 (adopted from GPCRdb numbering ${ }^{29}$, the second residue after the family-wide conserved cysteine, 45.50, in ECL2) are Glu/Asp (e.g., GHRHR and PTH1R), ECL2 shows compact contacts with peptides through direct salt bridge (GHRHR: $\operatorname{Arg}^{11 \mathrm{P}}$ and D274 ${ }^{45.52 \mathrm{~b}}$ ), or intra-receptor salt bridge (PTH1R: K240 $2.67 \mathrm{~b}$ and D353 ${ }^{45.52 \mathrm{~b}}$ ). In the case of GCGR/ GLP-1R whose residues at 45.52 are Thr, ECL2 of GCGR forms multiple hydrogen bonds with glucagon via Gly ${ }^{4 \mathrm{P}}, \mathrm{Ser}^{8 \mathrm{P}}$, and $\mathrm{Ser}^{11 \mathrm{P}}$, and ECL2 of GLP-1R forms polar interaction with multiple serines (Ser ${ }^{14 \mathrm{P}}$, Ser $^{17 \mathrm{P}}$, and Ser ${ }^{18 \mathrm{P}}$ ) of GLP-1 via T298 ${ }^{45.52 \mathrm{~b}}$ and electrostatic interaction with $\mathrm{His}^{7 \mathrm{P}}$ via inward chain of R299ECL2. Remarkably, the binding of ExP5 induces reorganization of ECL2-peptide interface, where R299ECL2 rotates outward and forms salt bridges with $\mathrm{Glu}^{16 \mathrm{P}}$.

G protein coupling by GHRHR. Like other class B GPCRs, the outward movement of the cytoplasmic end of TM6, and concomitant shift of TM5 and ICL3 of GHRHR form a cavity to accommodate a 5 helix of $\mathrm{Ga}_{\mathrm{s}}$. This process also involves TM2, TM3, ICL2, and helix 8. The interface residues in this cavity are highly conserved among class B GPCRs, and the arrangement of GHRHR- $\mathrm{G}_{\mathrm{s}}$ complex is also similar to other class B GPCR-G complexes, and follows a common mechanism of $G$ protein coupling. The high-resolution cryo-EM map allows us unambiguously to assign the water molecules between the interface of GHRHR and $\mathrm{Ga}_{\mathrm{s}}$. Comparing with other class B GPCR-G complexes in which a5 helix of $\mathrm{Ga}_{\mathrm{s}}$ loosely interacts with the receptor TM7-H8 hinge (Supplementary Fig. 9a, b), we found two water molecules in the GHRH-GHRHR-G $\mathrm{G}_{\mathrm{s}}$ complex establishing extensive polar interaction network connecting the $\mathrm{C}$-terminus of $\mathrm{Ga}_{\mathrm{s}}$ to the TM7-H8 hinge of GHRHR, thereby causing considerable conformational changes compared to GLP-1R-G and PTH1R-G s $_{\mathrm{s}}$ structures (Supplementary Fig. 9c, d). Specifically, the crevice between the TM7-H8 hinge and TM6 in GHRHR-G complex is broader than that in the PTH1R-G and GLP-1R-G complexes, which may be responsible for $G$ protein moving $3 \AA$ toward the cytoplasmic core of the TMD bundle (Supplementary Fig. 9a, b).

Besides contacting the cytoplasmic cavity of TMs 2, 3, 6, and 7 induced by the opening of the cytoplasmic half of TM6 via $\mathrm{Ga}_{\mathrm{s}}$, 
a

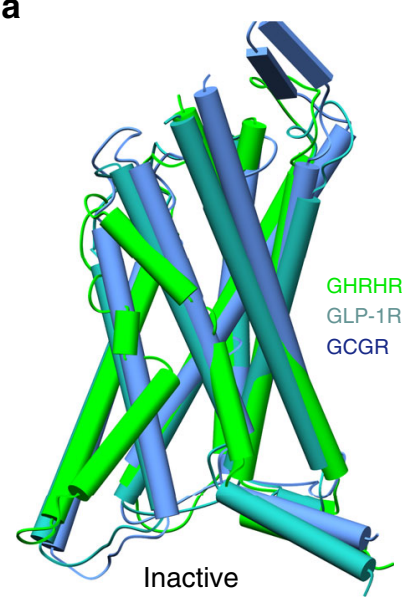

b

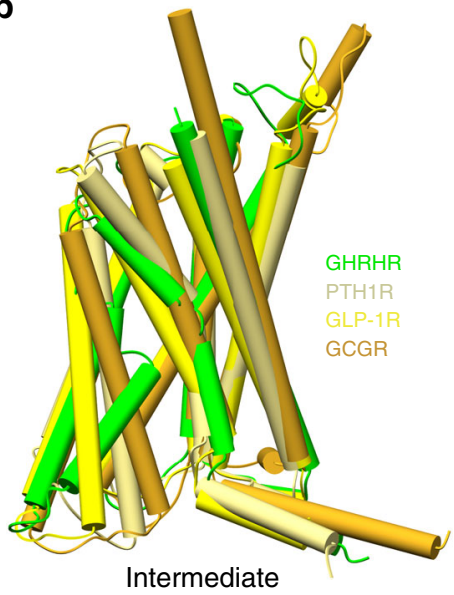

C

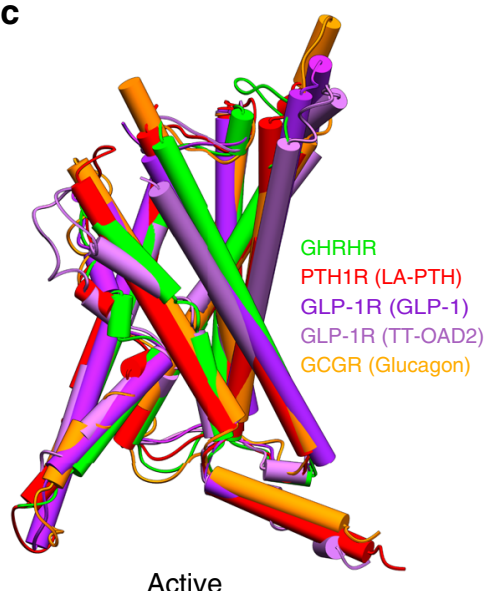

d

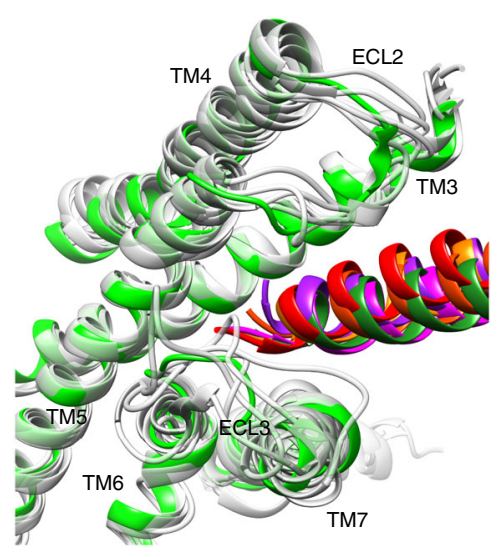

GHRH (GHRHR) LA-PTH (PTH1R) PACAP38 (PAC1B) Glucagon (GCGR) GLP-1 (GLP-1R) ExP5 (GLP-1R)
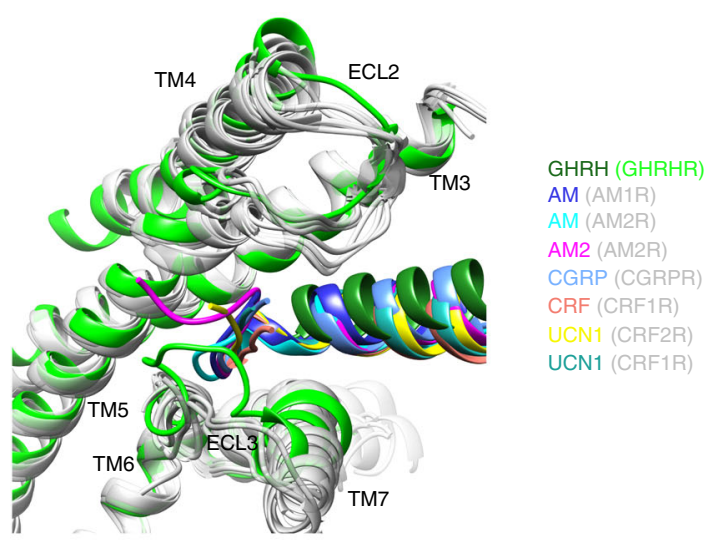

e
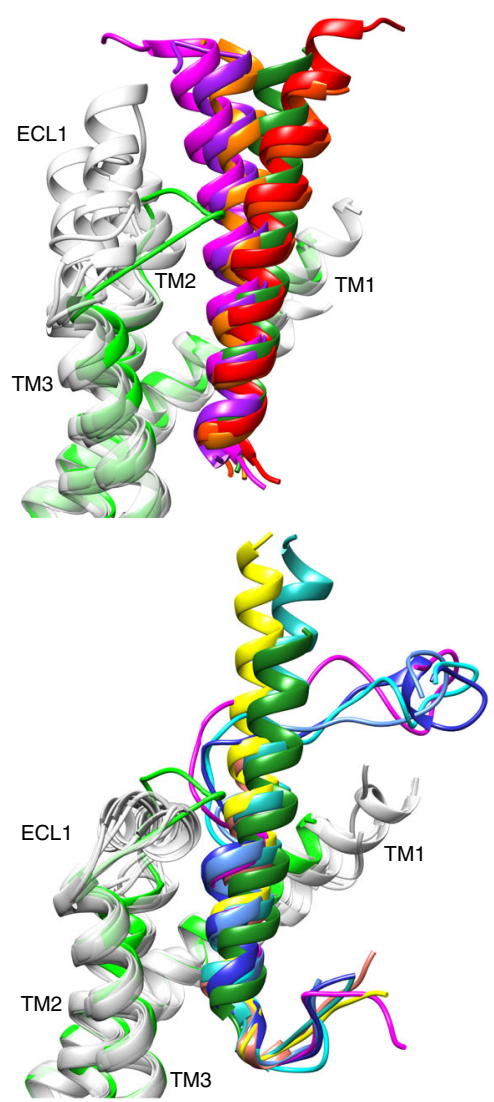

Fig. 3 Structural comparison of active GHRHR with other class B GRCRs in active, intermediate, and inactive states. a-c Comparison of active GHRHR with inactive (a), agonist-bound intermediate (b), and both agonist- and G-protein-bound active (c) class B GPCRs. d, e Comparison of ligand conformations in G-protein-bound class B GPCRs; close-up view of the N-terminus (d), middle region (e), and C-terminus (e) of bound peptides in class B GPCRs.

heterotrimeric $\mathrm{G}_{\mathrm{s}}$ protein also establishes an electrostatic interaction network with ICL1 and H8 via G $\beta$ (Supplementary Fig. 9e). Specifically, four residues (D312 of G 3, R156 $12.49 \mathrm{~b}$ of ICL1, and E386 $8.53 \mathrm{~b}$ and R389.56b of $\mathrm{H} 8$ ) are clustered at the interface of GHRHR-G $\beta$ by forming salt bridges. Such an organization is conserved across class B GPCRs, evidenced by the highly conserved residues and previous studies on GLP-1R and PTH1R. Notably, an additional component, Arg/Lys ${ }^{8.60 b}$, from GLP-1R, GCGR, and PTH1R may join and further stabilizes the network, while G3938.60b of GHRHR has neglectable contacts with $G \beta$. Indeed, $G 393^{8.60 b} \mathrm{R}$ enhances the potency of $\mathrm{GHRH}$, probably by strengthening the electrostatic interactions between GHRHR and $G_{s}$, whereas the diminished potency of a double mutant $\left(\mathrm{R} 156^{12.49 \mathrm{~b}} \mathrm{~A} / \mathrm{R} 389^{8.56 \mathrm{~b}} \mathrm{~A}\right)$ is likely resulted from the disruption of the electrostatic interaction network (Supplementary Fig. 9f).

Implication of disease-causing mutations. Based on the structure information, 25 missense mutants (21 were reported to be linked with IGHD previously; Fig. 4a and Supplementary Table 5) were made and assessed for their effects on cAMP signaling and $\beta$-arrestin 2 recruitment. Four of them were further analyzed by 
a

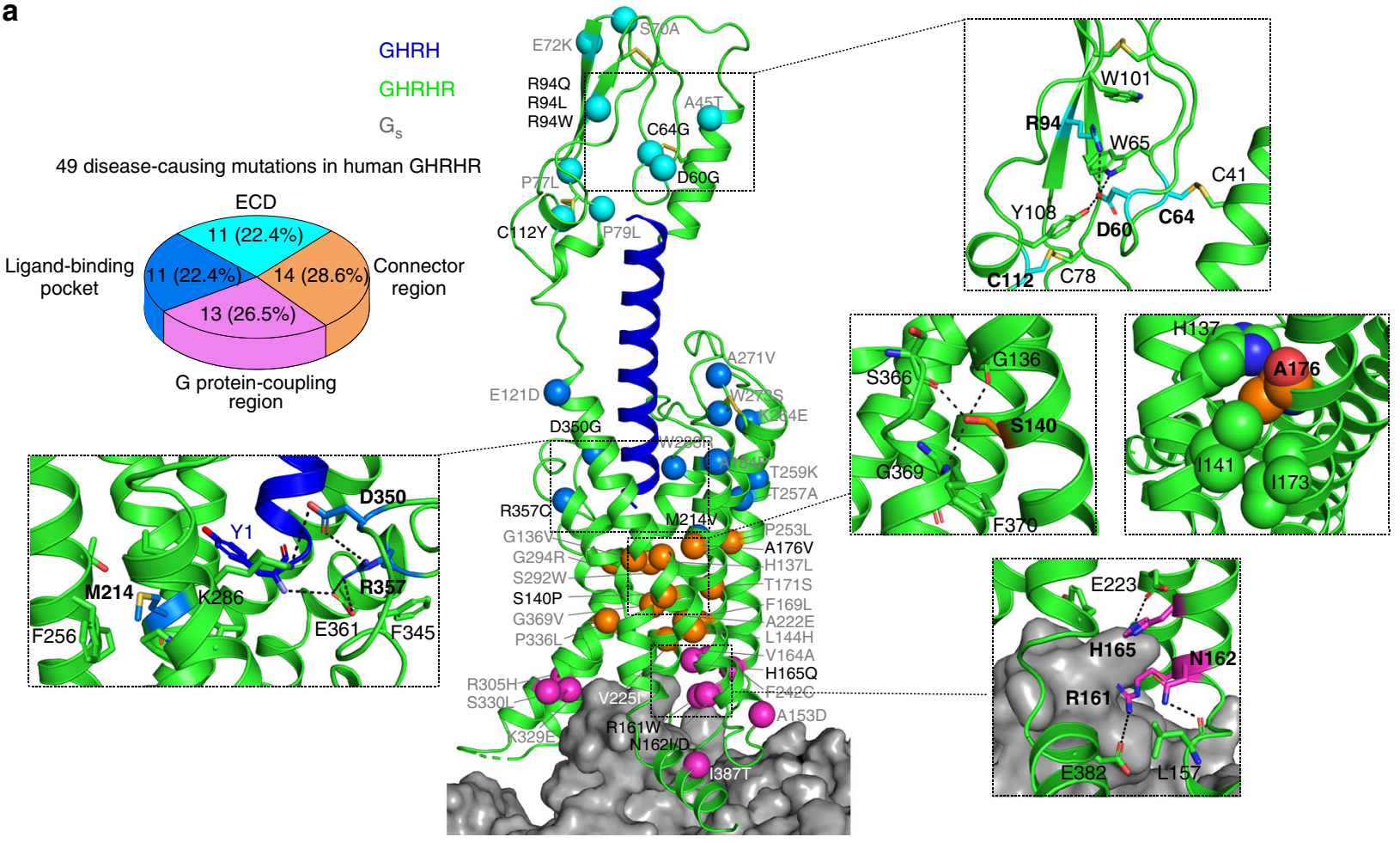

b
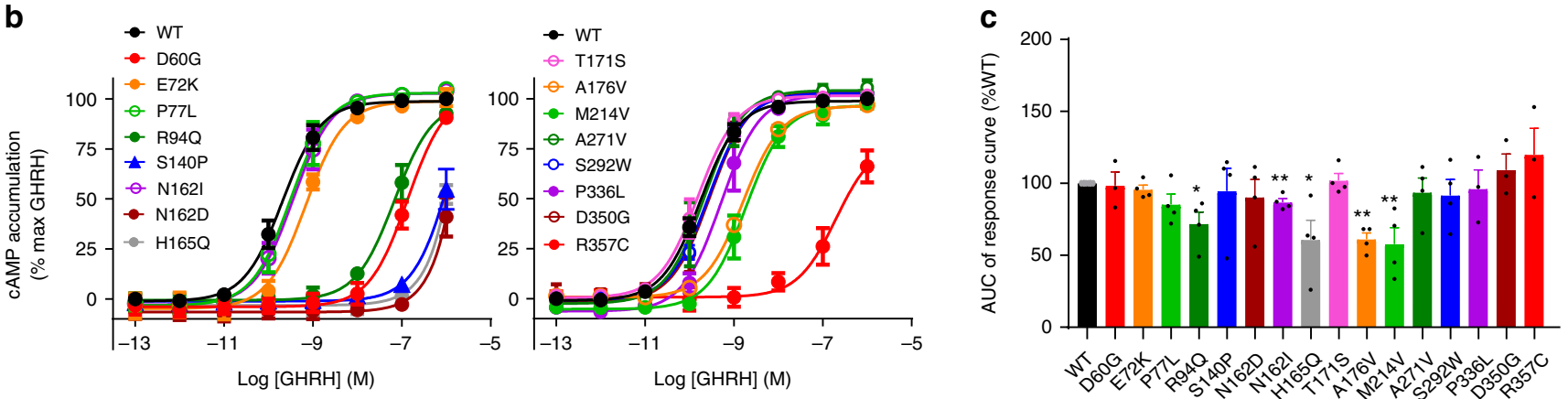

Fig. 4 Disease-causing missense mutations of GHRHR. IGHD refers to conditions of growth hormone deficiency that are not necessarily associated with other pituitary hormone deficiencies or with an organic lesion. While there is no single cause identified for IGHD, defects in growth hormone, GHRHR and $\mathrm{GH} 1$ genes are implicated as causative factors ${ }^{55}$. Of which, naturally occurring missense mutations of GHRHR have been studied extensively ${ }^{56}$. a Naturally occurring mutations in the ECD, ligand-binding pocket, and G-protein-coupling region are colored cyan, marine, and magenta, respectively. The central region connecting the ligand-binding pocket and G-protein-coupling region is colored orange. b, c Functional assessment of effects of disease-causing GHRHR mutations on $\mathrm{G}_{\mathrm{s}}$-mediated $\mathrm{cAMP}$ accumulation $(\mathbf{b})$ and $\beta$-arrestin2 recruitment (c), cAMP accumulation assays and $\beta$-arrestin2 recruitment were performed in HEK 293 T cells and data shown are means \pm S.E.M. of at least three independent experiments $(n=3-8)$, performed in quadruplicate or duplicate, respectively. Statistically significant differences were determined with a two-tailed Student's $t$ test. ${ }^{\star} P<0.05$, ${ }^{\star \star} P<0.01$ vs. wild type (WT); AUC area-under-the-curve. Source data are provided as a Source data file.

MD simulations. It is established that a common fold of threelayer $\alpha-\beta-\beta / \alpha$ architecture ${ }^{30,31}$ across class B GPCRs is stabilized by three conserved interlayer disulfide bonds (C41-C64, C55C96, and $\mathrm{C} 78-\mathrm{C} 112$ ) and the salt bridge/pi stacking within the family-wide conserved D-W-R/K-W motif (D60-W65-R94W101) in GHRHR, that connect two $\beta$-sheets to directly interact with the peptide ligands. Presumably, naturally occurring mutations may disrupt the disulfide bonds (C64G and C112Y) or the conserved D-W-R/K-W motif (R94Q/L/W), thereby destabilizing the stability of ECD and preventing GHRH recognition. Indeed, our MD simulation and functional studies suggest that the IGHD-associated mutation $\mathrm{R}_{4} \mathrm{Q}^{32}$ breaks the salt bridge with D60, increases the flexibility of the ECD, decreases the area of GHRH-GHRHR interface, and reduces GHRH-induced cAMP accumulation (Fig. 4b and Supplementary Fig. 10a). We then examined if mutations on D60 could also eliminate the electrostatic interaction between D60 and R94 to exhibit similar functional and phenotypical outcomes. D60G is known for the little mouse phenomenon-the first naturally occurring animal model of inherited autosomal recessive $\mathrm{GH}$-deficient dwarfism ${ }^{33}$. Like R94Q, it reduces GHRH binding affinity ${ }^{34,35}$, diminishes its potency on cAMP accumulation (Fig. 4b), and weakens GHRH binding in MD simulations (Supplementary Fig. 10a).

In the peptide-binding pocket, R357C was shown to loosen the compact GHRHR contacts in MD simulation (Supplementary Fig. 10b) and reduce GHRH potency by 1000-fold (Fig. 4b), consistent with the decreased potency of R357A and D350A mutants (Fig. 2d). Different from Ala/Val/Val in GLP-1R/PTH1R/GCGR, the 
residue at 3.41 of GHRHR located at the bottom of the ligandbinding pocket is M214, whose long side chain points to a hydrophobic cleft between TM4 and TM5. Another mutation associated with IGHD, M214V, was found to decrease GHRH potency by tenfold and selectively reduce $\beta$-arrestin 2 recruitment (Fig. 4b).

The connector region is known for the sharp kink in the middle of TM6 around Pro ${ }^{6.47 b}-\mathrm{X}-\mathrm{X}-\mathrm{Gly}{ }^{6.50 \mathrm{~b}}$. It appears that $\mathrm{P} 336^{6.47 \mathrm{~b}} \mathrm{~L}$ hampered the sharp kink upon receptor activation evidenced by a reduced $\mathrm{EC}_{50}$ value for cAMP signaling (Fig. 4b). As a comparison, $\mathrm{S} 140^{1.50 \mathrm{~b}} \mathrm{P}$ eliminated the hydrogen bonds between TM1 and TM7, increased the flexibility of TMD region and the bound GHRH, and reduced cAMP accumulation by over 7000fold (Fig. 4b and Supplementary Fig. 10b). A1762.61b oriented toward TM1, and contributed close hydrophobic contacts with

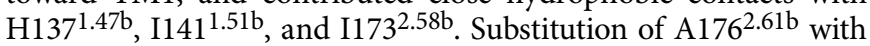
a larger hydrophobic valine at this position reduced GHRH potency (tenfold) and $\beta$-arrestin2 recruitment (39\%).

Within the G-protein-coupling region, $\mathrm{N} 162^{2.47 \mathrm{~b}} \mathrm{I}, \mathrm{N} 162^{2.47 \mathrm{~b}} \mathrm{D}$, and $H 165^{2.50 b} \mathrm{Q}$ were previously proposed to be deleterious ${ }^{36}-\mathrm{a}$ view that was verified experimentally in this study (Fig. 4b) As shown in the structure (Fig. 4a), N162 formed a hydrogen bond with the backbone of L157 ICL1, H165 ${ }^{2.50 \mathrm{~b}}$ from HETX motif ${ }^{10,14}$ played a crucial role in receptor activation, while H165Q might have directly altered receptor- $G$ protein interface and abolished $\mathrm{G}$ protein coupling.

\section{Discussion}

In summary, the high-resolution structure of the GHRHR- $\mathrm{G}_{\mathrm{s}}$ complex, and mutational studies provide a basis for GHRH recognition and receptor activation. These results also unveiled three distinct malfunctioning mechanisms of GHRHR signaling: (i) impaired peptide binding in the ECD (D60G and R94Q); (ii) reduced ligand recognition (R357C)/G protein coupling (N162I/ $\mathrm{D}$ and $\mathrm{H} 165 \mathrm{Q}$ via functional validation); and (iii) disrupted signal propagation in the connector region (S140P). Together, our work solved a longstanding puzzle of IGHD-causing mutations, which result in the impairment of GHRHR-mediated signaling at the ECD or TMD, singly or in combination.

\section{Methods}

Cell culture. Spodoptera frugiperda (Sf9) insect cells (Expression Systems) were grown in ESF 921 serum-free medium (Expression Systems) at $27^{\circ} \mathrm{C}$ and 120 r.p. $\mathrm{m}$. HEK 293 T cells were purchased from the Cell Bank at the Chinese Academy of Sciences, cultured in Dulbecco's modified Eagle's medium (DMEM; Life Technologies) supplemented with $10 \%$ fetal bovine serum (Gibco) and maintained in a humidified chamber with $5 \% \mathrm{CO}_{2}$ at $37^{\circ} \mathrm{C}$

Constructs of $\mathbf{G H R H R}$ and $\mathbf{G}_{\mathbf{s}}$ heterotrimer. For structural studies, wild-type (WT) human GHRHR DNA (Genewiz) was cloned into pFastBac vector (Invitrogen) with its native signal sequence (M1-G22) replaced by the hemagglutinin (HA) signal peptide. Eighteen amino acids (A406-C423) were truncated at the Cterminus and $\mathrm{LgBiT}$ subunit (Promega) was fused with a 15 -amino acid polypeptide (GSSGGGGSGGGGSSG) linker at the C-terminus followed by a Tev protease cleavage site and a double maltose-binding protein (MBP) tag to facilitate expression and purification. A dominant-negative human $\mathrm{Ga}_{\mathrm{s}}\left(\mathrm{DNGa}_{\mathrm{s}}\right)$ was generated by site-directed mutagenesis as previously described to stabilize the interaction with the $\beta \gamma$ subunits ${ }^{11,37}$. Rat $\mathrm{G} \beta 1$ with an $\mathrm{N}$-terminal $10 \times$ His-tag was fused with a $\mathrm{SmBiT}^{38}$ (peptide 86, Promega) subunit by the 15 -amino acid polypeptide linker at its C-terminus. Human DNG $\alpha_{\mathrm{s}}$, rat $\mathrm{G} \beta 1$, and bovine $\mathrm{G} \gamma 2$ were cloned into pFastBac vector, respectively. In addition to clone the constructs into the pBiT vector (Promega) for NanoBiT assay, they all contained an $\mathrm{N}$-terminal Flag tag (DYKDDDD) proceeded by a HA signal sequence, and were cloned into the pcDNA3.1 vector (Invitrogen) for functional studies.

To obtain a GHRHR- $\mathrm{G}_{\mathrm{s}}$ complex with good homogeneity and stability, the human GHRHR was modified by replacing the $\mathrm{N}$-terminal native signal peptide with HA sequence. In addition, 18 residues (A406-C423) were truncated at the Cterminus, followed by an LgBiT subunit and a double MBP affinity tag to improve protein yield and stability (Supplementary Fig. 2a). These modifications did not affect ligand binding and receptor activation (Supplementary Fig. 2b, c and Supplementary Table 1). In addition, a DNGa ${ }_{s}^{11,37}$, His10-G $\beta 1$-peptide 86 and
G $\gamma 2$ were co-expressed with GHRHR(23-405)-15AA-LgBiT-2MBP in insect cells. Formation of GHRHR- $\mathrm{G}_{\mathrm{s}}$ complex on the membrane was stimulated with an excess amount of GHRH, and in the presence of $\mathrm{Ga}$ - and G $\beta$-binding nanobody $35(\mathrm{Nb} 35)^{39}$ (Supplementary Fig. 2d-e).

Expression and purification of GHRHR-G $_{\mathbf{s}}$ complex. GHRHR-15AA-LgBiT2MBP or GHRHR-2MBP, DNG $\alpha_{\mathrm{s}}$, His10-G $\beta 1$-peptide 86, and G $\gamma 2$ recombinant baculoviruses were prepared using Bac-to-Bac Baculovirus Expression System (Invitrogen) severally. Sf9 insect cells were grown to a density of $3 \times 10^{6}$ cells per $\mathrm{mL}$ and then coinfected with four separate viruses at a ratio of 1:3:3:3 for GHRHR, $D_{N G a}, G \beta 1$, and $G \gamma 2$. Cells were harvested by centrifugation $48 \mathrm{~h}$ post infection and pellets were stored at $-80^{\circ} \mathrm{C}$ until use.

The cell pellets were thawed on ice and lysed in a buffer containing $20 \mathrm{mM}$ HEPES, pH 7.5, $100 \mathrm{mM} \mathrm{NaCl}, 10 \%$ (v/v) glycerol, $10 \mathrm{mM} \mathrm{MgCl}_{2}, 5 \mathrm{mM} \mathrm{CaCl}_{2}$, $1 \mathrm{mM} \mathrm{MnCl} 2,100 \mu \mathrm{M}$ TCEP, and supplemented with EDTA-free protease inhibitor cocktail (Bimake) by dounce homogenization. The complex formation

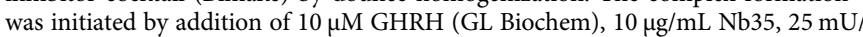
$\mathrm{mL}$ apyrase (NEB), and the lysate was incubated for $1.5 \mathrm{~h}$ at room temperature (RT). The membrane was further solubilized by $0.5 \%(\mathrm{w} / \mathrm{v})$ lauryl maltose neopentyl glycol (LMNG; Anatrace) and $0.1 \%(\mathrm{w} / \mathrm{v})$ cholesterol hemisuccinate (CHS; Anatrace) for $2 \mathrm{~h}$ at $4{ }^{\circ} \mathrm{C}$. After centrifugation at $65,000 \times g$ for $30 \mathrm{~min}$, the supernatant was isolated and incubated with amylose resin (NEB) for $2 \mathrm{~h}$ at $4{ }^{\circ} \mathrm{C}$. The resin was then collected by centrifugation at $600 \times g$ for 10 min, loaded into a gravity flow column (Sangon Biotech), and first washed with five column volumes of buffer containing $20 \mathrm{mM}$ HEPES, pH 7.5, $100 \mathrm{mM} \mathrm{NaCl}, 10 \%$ (v/v) glycerol, $5 \mathrm{mM} \mathrm{MgCl}, 1 \mathrm{mM} \mathrm{MnCl} 2,25 \mu \mathrm{M}$ TCEP, $1 \mu \mathrm{M}$ GHRH, 0.1\% (w/v) LMNG, and $0.02 \%$ (w/v) CHS, followed by washing with 15 column volumes of buffer containing $20 \mathrm{mM}$ HEPES, pH 7.5, $100 \mathrm{mM} \mathrm{NaCl}, 10 \%(\mathrm{v} / \mathrm{v})$ glycerol, $5 \mathrm{mM}$ $\mathrm{MgCl}_{2}, 1 \mathrm{mM} \mathrm{MnCl} 2,25 \mu \mathrm{M}$ TCEP, $1 \mu \mathrm{M} \mathrm{GHRH}, 0.03 \%$ (w/v) LMNG, $0.01 \%$ (w/v) glyco-diosgenin (Anatrace), and $0.008 \%(\mathrm{w} / \mathrm{v}) \mathrm{CHS}$. The protein was then incubated overnight with His-tagged Tev protease (customer-made) on the column to remove the C-terminal 2MBP-tag in the buffer above. The flow through was collected next day and concentrated with a $100 \mathrm{kDa}$ molecular weight cut-off concentrator (Millipore). Concentrated GHRH-GHRHR- $\mathrm{G}_{\mathrm{s}}-\mathrm{Nb} 35$ complex was loaded onto a Superdex 200 increase 10/300 GL column (GE Healthcare) with running buffer containing $20 \mathrm{mM}$ HEPES, pH 7.5, $100 \mathrm{mM} \mathrm{NaCl}, 10 \mathrm{mM} \mathrm{MgCl} 2$ $100 \mu \mathrm{M}$ TCEP, $5 \mu \mathrm{M} \mathrm{GHRH}$, and $0.001 \%$ digitonin (Anatrace). The fractions for monomeric complex were collected and concentrated to $20-30 \mathrm{mg} / \mathrm{mL}$ for EM examination.

Expression and purification of $\mathbf{N b 3 5}$. Nb35 with a C-terminal $6 \times$ His-tag was expressed in the periplasm of Escherichia coli BL21 (DE3) cells, extracted and purified by nickel affinity chromatography as previously described ${ }^{36}$. Eluted protein was concentrated using a $10 \mathrm{kDa}$ molecular weight cut-off concentrator (Millipore) and loaded onto a HiLoad 16/600 Superdex 75 column (GE Healthcare), with running buffer containing $20 \mathrm{mM}$ HEPES, $\mathrm{pH} 7.5$, and $100 \mathrm{mM} \mathrm{NaCl}$ The monomeric fractions were pooled and supplemented with $30 \%(\mathrm{v} / \mathrm{v})$ glycerol. Purified Nb35 was finally flash frozen in liquid nitrogen and stored in $-80^{\circ} \mathrm{C}$.

\section{Cryo-EM data acquisition and image processing. The purified}

GHRH-GHRHR-G s $_{\text {s }}$ complex $(3.5 \mu \mathrm{L})$ at a concentration of $22 \mathrm{mg} / \mathrm{mL}$ was applied to glow-discharged holey carbon grids (Quantifoil R1.2/1.3, 200 mesh), and subsequently vitrified using a Vitrobot Mark IV (ThermoFisher Scientific). Cryo-EM images were collected on a Titan Krios equipped with a Gatan K2 Summit direct electron. The microscope was operated at $300 \mathrm{kV}$ accelerating voltage, at a nominal magnification of $29,000 \times$ in counting mode, corresponding to a pixel size of 1.014 $\AA$ A. In total, 3813 image stacks were obtained at the dose rate of $\sim 7.8$ electrons per $\AA^{2}$ per second with a defocus range of -0.5 to $-2.5 \mu \mathrm{m}$. The total exposure time was set to $8 \mathrm{~s}$ with intermediate frames recorded every $0.2 \mathrm{~s}$, resulting in an accumulated of dose of 62 electrons per $\AA^{2}$.

Dose-fractionated image stacks were subjected to beam-induced motion correction and dose-weighting using MotionCor2.1 (ref. ${ }^{40}$ ). A sum of all frames, filtered according to the exposure dose, in each image stack was used for further processing. Contrast transfer function parameters for each micrograph were determined by Gctf vl.06 (ref. ${ }^{41}$ ). Further data processing was performed in RELION-3.0-beta2 (ref. ${ }^{42}$ ). Particle selection, two-dimensional classification, and the first round of three-dimensional classification were performed on a binned dataset with a pixel size of $2.028 \AA$

Auto-picking yielded 2,586,606 particle projections that were subjected to reference-free two-dimensional classification to discard false-positive particles or particles categorized in poorly defined classes, producing $1,456,108$ projections for further processing. This subset of particle projections was subjected to consecutive rounds of three-dimensional classification with a pixel size of $2.028 \AA$. The map of PTH1R-G G $_{\text {s }}$ complex (EMDB-0410) low-pass filtered to $40 \AA$ was used as an initial reference model for two rounds of three-dimensional classification, resulting in two subsets accounting for 481,220 projections that showed better EM densities. Further three-dimensional classifications focusing on the complex, with the exception of AHD of Gas, produced one good subset with higher resolution, which was subsequently subjected to three-dimensional refinement and Bayesian 
polishing. The final refinement with frames 1-25 generated a map with an indicated global resolution of $2.6 \AA$, with 307,018 projections at a Fourier shell correlation of 0.143 . Local resolution was determined using the Bsoft package with half maps as input maps ${ }^{43}$.

Model building and refinement. The structure of the LA-PTH-PTH1R-G complex was used as an initial template for model building. Lipid coordinates and geometry restraints were generated using phenix.elbow. Models were docked into the EM density map using UCSF Chimera ${ }^{44}$. This starting model was then subjected to iterative rounds of manual adjustment and automated refinement in $\mathrm{Coot}^{45}$ and Phenix ${ }^{46}$, respectively. The final refinement statistics were validated using the module "comprehensive validation (cryo-EM)" in PHENIX. Structural figures were prepared in Chimera, Chimera X, and PyMOL (https://pymol.org/2/). The final refinement statistics are provided in Supplementary Table 2.

NanoBiT assay. HEK $293 \mathrm{~T}$ cells were transiently transfected with GHRHR-LgBiT, $\mathrm{Ga}_{\mathrm{s}}, \mathrm{G} \beta 1$-SmBiT (peptide 86 or 114), and $\mathrm{G} \gamma 248 \mathrm{~h}$ before assaying in a mass ratio of 1:1:1:1. Twenty-four hour post transfection, cells were digested and seeded into 96-well white plate (PerkinElmer) at optimal density (30,000 cells/well) and the medium was replaced with Opti-MEM (Gibco) $3 \mathrm{~h}$ prior to assaying. After equilibration to ambient temperature, the Nano-Glo Live Cell Substrate (Promega) was 20 -fold diluted to create a $5 \times$ stock and mixed with cell culture medium to a final $1 \times$ concentration, then luminescence signal was measured using an EnVision Multimode Plate Reader (PerkinElmer). Following the baseline reading at $10 \mathrm{~s}$ interval for $2.5 \mathrm{~min}$, GHRH was diluted with Opti-MEM and added to a final concentration of $10 \mathrm{nM}$, and reading continued at $10 \mathrm{~s}$ intervals for $10 \mathrm{~min}$. RLU time-course response curves were normalized to that of background and calculated as percentage of the baseline value. Interaction intensity of the complex was expressed as area-under-the-curve (AUC) across the full kinetic trace (0-10 min).

Negative-stain EM. Uranyl formate (0.75\% (w/v), Electron Microscopy Sciences) solution was prepared as previously described ${ }^{47}$. Copper grids $(300$ mesh) coated with carbon film (Electron Microscopy Sciences) were glow charged, using PELCO easiGlow ${ }^{\mathrm{TM}}$ Glow Discharge Cleaning System (Ted Pella Inc.) for $25 \mathrm{~s}$ at $25 \mathrm{~mA}$. Purified protein samples $(4 \mu \mathrm{L}, 0.01 \mathrm{mg} / \mathrm{mL})$ were applied to glow-charged holey grids for $1 \mathrm{~min}$ and then blotted off using filter paper. Uranyl formate solution $(0.75 \%, 5 \mu \mathrm{L})$ was added to the grid surface twice: the first application was blotted off immediately and the second was retained for $1 \mathrm{~min}$ before blotting with filter paper. The stained girds were loaded into a Tecnai G2 Spirit transmission electron microscope (Thermo FEI) operated at $120 \mathrm{kV}$. Negative staining images were acquired at a magnification of $57,000 \times$ within a -1.5 to $-2.0 \mu \mathrm{m}$ defocus range.

Dynamic light scattering. Dynamic light scattering determines size and size distribution by measuring the rapid changes in laser light intensity of molecules or particles in solution. Briefly, freshly purified protein samples $(15 \mu \mathrm{L}$ each) were concentrated to $1 \mathrm{mg} / \mathrm{mL}$ and loaded into DynaPro NanoStar (Wyatt Technology) to measure time-dependent fluctuations of scattered intensity at $30^{\circ} \mathrm{C}$. Data were analyzed using the dynamics software supplied with the instrument.

cAMP accumulation assay. GHRH-stimulated cAMP accumulation was measured by a LANCE Ultra cAMP kit (PerkinElmer). Briefly, $24 \mathrm{~h}$ after transfection with GHRHR(23-423), GHRHR(23-405), GHRHR(23-405)-LgBiT-2MBP, or HAFlag-GHRHR (WT and mutants), HEK $293 \mathrm{~T}$ cells (3000/well in 384-well white plates, PerkinElmer) were digested by $0.2 \%(\mathrm{w} / \mathrm{v})$ EDTA and $5 \mu \mathrm{L}$ stimulation buffer (HBSS supplemented with $5 \mathrm{mM}$ HEPES, $0.5 \mathrm{mM} \mathrm{IBMX}$, and $0.1 \%(\mathrm{w} / \mathrm{v})$ BSA, pH 7.4). Different concentrations $(5 \mu \mathrm{L})$ of GHRH were then added and the stimulation lasted for $30 \mathrm{~min}$ at RT. The reaction was stopped by adding $5 \mu \mathrm{L}$ EucAMP tracer and $5 \mu \mathrm{L}$ ULight-anti-cAMP. After $1 \mathrm{~h} \mathrm{RT}$ incubation, TR-FRET signals (excitation: $320 \mathrm{~nm}$, emission: 615 and $665 \mathrm{~nm}$ ) were measured by an EnVision (PerkinElmer).

Whole cell binding assay. This assay was conducted in 96-well Iso-plates (PerkinElmer) coated with poly-D-lysine hydrobromide (Sigma-Aldrich). Twenty-four hour after transfection with GHRHR(23-423) or GHRHR(23-405)-LgBiT-2MBP, HEK $293 \mathrm{~T}$ cells were washed twice, and incubated with blocking buffer (DMEM medium supplemented with $33 \mathrm{mM}$ HEPES, and $0.1 \%$ (w/v) BSA, pH 7.4) for $2 \mathrm{~h}$ at $37^{\circ} \mathrm{C}$. Then, radiolabeled ${ }^{125} \mathrm{I}-\mathrm{GHRH}(80 \mathrm{pM}$, Phoenix Biotech) and seven decreasing concentrations of unlabeled peptide $(10 \mu \mathrm{M}$, five serial gradient dilutions) were added and competitively reacted with the cells in binding buffer (PBS supplemented with $10 \%(\mathrm{w} / \mathrm{v}) \mathrm{BSA}, \mathrm{pH}$ 7.4) at RT for $3 \mathrm{~h}$. Cells were washed with ice-cold PBS and lysed by $50 \mu \mathrm{L}$ lysis buffer (PBS supplemented with $20 \mathrm{mM}$ Tris$\mathrm{HCl}$ and $1 \%(\mathrm{v} / \mathrm{v})$ Triton X-100, pH 7.4). Finally, $150 \mu \mathrm{L}$ of scintillation cocktail (OptiPhase SuperMix, PerkinElmer) was employed and radioactivity (counts per minute) read in a scintillation counter (MicroBeta ${ }^{2}$ plate counter, PerkinElmer).

$\boldsymbol{\beta}$-arrestin2 recruitment assay. HEK $293 \mathrm{~T}$ cells were seeded at a density of 30,000 cells per well into 96-well culture plates pretreated with poly-D-lysine hydrobromide. After incubation for $24 \mathrm{~h}$ to reach $70-80 \%$ confluence, the cells were transiently transfected with HA-Flag-GHRHR-Rluc8 (WT and mutants) and $\beta$-arrestin2-Venus at a 1:9 mass ratio, using Lipofectamine 3000 reagent (Invitrogen) and cultured for another $24 \mathrm{~h}$. Thereafter, cells were washed once and incubated for $30 \mathrm{~min}$ at $37^{\circ} \mathrm{C}$ with HBSS buffer ( $\mathrm{pH} \mathrm{7.4)}$ supplemented with $0.1 \%$ $(\mathrm{m} / \mathrm{v}) \mathrm{BSA}$ and $10 \mathrm{mM}$ HEPES. A total of $5 \mu \mathrm{M}$ coelenterazine $\mathrm{h}$ (YEASEN Biotechnology) was then added and incubated for $5 \mathrm{~min}$ in the dark. The bioluminescence resonance energy transfer (BRET) signals were detected with an EnVision by calculating the ratio of emission at $535 \mathrm{~nm}$ over emission at $470 \mathrm{~nm}$. A $1.5 \mathrm{~min}$ baseline of BRET measurements was taken before the addition of $250 \mu \mathrm{M}$ GHRH or vehicle and BRET signal was measured at $10 \mathrm{~s}$ intervals for further $9 \mathrm{~min}$. After removing baseline and background readings by subtracting average values of the baseline measurement and average values of vehicle-treated samples, respectively, the AUC across the time-course response curve was determined and normalized to the WT, which was set to $100 \%$.

Molecular dynamics simulation. All-atom MD simulations of the GHRH-GHRHR were performed by Gromacs 2018.5. After removing all G protein subunits and heteroatoms, the receptor and its disease-causing mutants (D60G, R94Q, S140P, and R357C) were prepared and capped by the Protein Preparation Wizard (Schrodinger 2017-4). Titratable residues were left in their dominant state at $\mathrm{pH}$ 7.0. The complexes were embedded in a bilayer composed of 225 POPC lipids and solvated with $0.15 \mathrm{M} \mathrm{NaCl}$ in explicitly represented waters, using CHARMM-GUI Membrane Builder ${ }^{48}$. The CHARMM36-CAMP force filed ${ }^{49}$ was adopted for protein, peptides, lipids, and salt ions, while the CHARMM TIP3P model was chosen for water. The particle mesh Ewald method ${ }^{50}$ was used to treat all electrostatic interactions beyond a cutoff of $10 \AA$ and the bonds involving hydrogen atoms were constrained using LINCS algorithm ${ }^{51}$. The constructed system was firstly relaxed using the steepest descent energy minimization, followed with slow heating of the system to $310 \mathrm{~K}$ with restraints. The restraints were reduced gradually over $50 \mathrm{~ns}$, with a simulation step of 1 fs. Finally, a $1 \mu \mathrm{s}$ production run without restraints was carried out for each simulation, with a time step of $2 \mathrm{fs}$ in the NPT ensemble at $310 \mathrm{~K}$ and 1 bar using the v-rescale thermostat ${ }^{52}$ and the semiisotropic Parrinello-Rahman barostat ${ }^{53}$, respectively. The last $500 \mathrm{~ns}$ trajectory was used for analysis, and the GHRH-GHRHR interface area was calculated by the program FreeSASA ${ }^{54}$, using the Sharke-Rupley algorithm with a probe radius of $1.2 \AA$.

Statistical analysis. All functional study data were analyzed using Prism 7 (GraphPad) and presented as means \pm S.E.M. from at least three independent experiments. Concentration-response curves were evaluated with a threeparameter logistic equation. The significance was determined with either two-tailed Student's $t$ test or one-way ANOVA, and $P<0.05$ was considered statistically significant.

Reporting summary. Further information on research design is available in the Nature Research Reporting Summary linked to this article.

\section{Data availability}

Data supporting the findings of this manuscript are available from the corresponding authors upon reasonable request. A reporting summary for this article is available as a Supplementary information file. The atomic coordinates and the electron microscopy maps have been deposited in the Protein Data Bank (PDB) under accession number PDB 7CZ5 and Electron Microscopy Data Bank (EMDB) accession number EMD-30505. Source data are provided with this paper.

Received: 4 June 2020; Accepted: 14 September 2020; Published online: 15 October 2020

\section{References}

1. Pandol, S. J., Seifert, H., Thomas, M. W., Rivier, J. \& Vale, W. Growth hormone-releasing factor stimulates pancreatic enzyme secretion. Science 225, 326-328 (1984)

2. Guillemin, R. et al. Growth hormone-releasing factor from a human pancreatic tumor that caused acromegaly. Science 218, 585-587 (1982).

3. Cohen, L. E., Hashimoto, Y., Zanger, K., Wondisford, F. \& Radovick, S. CREBindependent regulation by CBP is a novel mechanism of human growth hormone gene expression. J. Clin. Investig. 104, 1123-1130 (1999).

4. Granata, R. Peripheral activities of growth hormone-releasing hormone. J. Endocrinol. Investig. 39, 721-727 (2016).

5. Cai, R. et al. Synthesis of new potent agonistic analogs of growth hormonereleasing hormone (GHRH) and evaluation of their endocrine and cardiac activities. Peptides 52, 104-112 (2014).

6. Fridlyand, L. E., Tamarina, N. A., Schally, A. V. \& Philipson, L. H. Growth hormone-releasing hormone in diabetes. Front. Endocrinol. 7, 129 (2016). 
7. Shen, J. et al. Regulation of vascular calcification by growth hormone-releasing hormone and its agonists. Circ. Res. 122, 1395-1408 (2018).

8. Villanova, T. et al. Antagonists of growth hormone-releasing hormone (GHRH) inhibit the growth of human malignant pleural mesothelioma. Proc. Natl Acad. Sci. USA 116, 2226-2231 (2019).

9. Schally, A. V., Varga, J. L. \& Engel, J. B. Antagonists of growth-hormonereleasing hormone: an emerging new therapy for cancer. Nat. Clin. Pr. Endocrinol. Metab. 4, 33-43 (2008).

10. Zhang, Y. et al. Cryo-EM structure of the activated GLP-1 receptor in complex with a G protein. Nature 546, 248-253 (2017).

11. Liang, Y. L. et al. Phase-plate cryo-EM structure of a biased agonist-bound human GLP-1 receptor- $\mathrm{G}_{\mathrm{s}}$ complex. Nature 555, 121-125 (2018).

12. Liang, Y. L. et al. Cryo-EM structure of the active, $\mathrm{G}_{\mathrm{s}}$-protein complexed, human CGRP receptor. Nature 561, 492-497 (2018).

13. Liang, Y. L. et al. Phase-plate cryo-EM structure of a class B GPCR-G-protein complex. Nature 546, 118-123 (2017).

14. Qiao, A. et al. Structural basis of $\mathrm{G}_{\mathrm{s}}$ and $\mathrm{G}_{\mathrm{i}}$ recognition by the human glucagon receptor. Science 367, 1346-1352 (2020).

15. Zhao, L. H. et al. Structure and dynamics of the active human parathyroid hormone receptor-1. Science 364, 148-153 (2019).

16. Ma, S. et al. Molecular basis for hormone recognition and activation of corticotropin-releasing factor receptors. Mol. Cell 77, 669-680 e664 (2020).

17. Liang, Y. L. et al. Toward a structural understanding of class B GPCR peptide binding and activation. Mol. Cell 77, 656-668 e655 (2020).

18. Liang, Y. L. et al. Structure and dynamics of adrenomedullin receptors AM1 and AM2 reveal key mechanisms in the control of receptor phenotype by receptor activity-modifying proteins. ACS Pharm. Transl. Sci. 3, 263-284 (2020).

19. Duan, J. et al. Cryo-EM structure of an activated VIP1 receptor-G protein complex revealed by a NanoBiT tethering strategy. Nat. Commun. 11, 4121 (2020).

20. Zhao, L. H. et al. Differential requirement of the extracellular domain in activation of class B G protein-coupled receptors. J. Biol. Chem. 291, 15119-15130 (2016).

21. Wootten, D., Simms, J., Miller, L. J., Christopoulos, A. \& Sexton, P. M. Polar transmembrane interactions drive formation of ligand-specific and signal pathway-biased family B G protein-coupled receptor conformations. Proc. Natl Acad. Sci. USA 110, 5211-5216 (2013).

22. Cervini, L. A., Donaldson, C. J., Koerber, S. C., Vale, W. W. \& Rivier, J. E. Human growth hormone-releasing hormone hGHRH(1-29)- $\mathrm{NH}_{2}$ : systematic structure-activity relationship studies. J. Med. Chem. 41, 717-727 (1998).

23. Matsoukas, M. T. \& Spyroulias, G. A. Dynamic properties of the growth hormone releasing hormone receptor (GHRHR) and molecular determinants of GHRH binding. Mol. Biosyst. 13, 1313-1322 (2017).

24. Zhang, H. et al. Structure of the glucagon receptor in complex with a glucagon analogue. Nature 553, 106-110 (2018).

25. Wootten, D. et al. The extracellular surface of the GLP-1 receptor is a molecular trigger for biased agonism. Cell 165, 1632-1643 (2016).

26. Song, G. et al. Human GLP-1 receptor transmembrane domain structure in complex with allosteric modulators. Nature 546, 312-315 (2017).

27. Siu, F. Y. et al. Structure of the human glucagon class B G-protein-coupled receptor. Nature 499, 444-449 (2013).

28. Thal, D. M., Glukhova, A., Sexton, P. M. \& Christopoulos, A. Structural insights into G-protein-coupled receptor allostery. Nature 559, 45-53 (2018).

29. Isberg, V. et al. Generic GPCR residue numbers-aligning topology maps while minding the gaps. Trends Pharm. Sci. 36, 22-31 (2015).

30. Hollenstein, K. et al. Insights into the structure of class B GPCRs. Trends Pharm. Sci. 35, 12-22 (2014).

31. Pal, K., Melcher, K. \& Xu, H. E. Structure and mechanism for recognition of peptide hormones by class B G-protein-coupled receptors. Acta Pharm. Sin. 33, 300-311 (2012)

32. Cohen, E. et al. Contribution of functionally assessed GHRHR mutations to idiopathic isolated growth hormone deficiency in patients without GH1 mutations. Hum. Mutat. 40, 2033-2043 (2019).

33. Eicher, E. M. \& Beamer, W. G. Inherited ateliotic dwarfism in mice. Characteristics of the mutation, little, on chromosome6. J. Hered. 67, 87-91 (1976).

34. Lin, S. C. et al. Molecular basis of the little mouse phenotype and implications for cell type-specific growth. Nature 364, 208-213 (1993).

35. Corazzini, V. \& Salvatori, R. Molecular and clinical aspects of GHRH receptor mutations. Endocr. Dev. 24, 106-117 (2013).

36. Landrum, M. J. et al. ClinVar: improving access to variant interpretations and supporting evidence. Nucleic Acids Res. 46, D1062-D1067 (2018).

37. Liang, $Y$. L. et al. Dominant negative $G$ proteins enhance formation and purification of agonist-GPCR-G protein complexes for structure determination. ACS Pharm. Transl. Sci. 1, 12-20 (2018).

38. Dixon, A. S. et al. NanoLuc complementation reporter optimized for accurate measurement of protein interactions in cells. ACS Chem. Biol. 11, 400-408 (2016).
39. Rasmussen, S. G. et al. Crystal structure of the $\beta_{2}$ adrenergic receptor- $\mathrm{G}_{\mathrm{s}}$ protein complex. Nature 477, 549-555 (2011)

40. Zheng, S. Q. et al. MotionCor2: anisotropic correction of beam-induced motion for improved cryo-electron microscopy. Nat. Methods 14, 331-332 (2017).

41. Zhang, K. Gctf: real-time CTF determination and correction. J. Struct. Biol. 193, 1-12 (2016).

42. Scheres, S. H. Processing of structurally heterogeneous cryo-EM data in RELION. Methods Enzymol. 579, 125-157 (2016).

43. Heymann, J. B. Single particle reconstruction and validation using Bsoft for the map challenge. J. Struct. Biol. 204, 90-95 (2018).

44. Pettersen, E. F. et al. UCSF Chimera-a visualization system for exploratory research and analysis. J. Comput. Chem. 25, 1605-1612 (2004).

45. Emsley, P. \& Cowtan, K. Coot: model-building tools for molecular graphics. Acta Crystallogr. D. Biol. Crystallogr. 60, 2126-2132 (2004).

46. Adams, P. D. et al. PHENIX: a comprehensive Python-based system for macromolecular structure solution. Acta Crystallogr. D. Biol. Crystallogr. 66, 213-221 (2010).

47. Booth, D.S., Avila-Sakar, A. \& Cheng, Y. Visualizing proteins and macromolecular complexes by negative stain EM: from grid preparation to image acquisition. J. Vis. Exp. 58, 3227 (2011).

48. Wu, E. L. et al. CHARMM-GUI membrane builder toward realistic biological membrane simulations. J. Comput. Chem. 35, 1997-2004 (2014).

49. Guvench, O. et al. CHARMM additive all-atom force field for carbohydrate derivatives and its utility in polysaccharide and carbohydrate-protein modeling. J. Chem. Theory Comput. 7, 3162-3180 (2011).

50. Darden, T., York, D. \& Pedersen, L. Particle mesh Ewald-an N.Log(N) method for Ewald sums in large systems. J. Chem. Phys. 98, 10089-10092 (1993).

51. Hess, B. P-LINCS: a parallel linear constraint solver for molecular simulation. J. Chem. Theory Comput. 4, 116-122 (2008).

52. Bussi, G., Donadio, D. \& Parrinello, M. Canonical sampling through velocity rescaling. J. Chem. Phys. 126, 014101 (2007).

53. Aoki, K. M. \& Yonezawa, F. Constant-pressure molecular-dynamics simulations of the crystal-smectic transition in systems of soft parallel spherocylinders. Phys. Rev. A 46, 6541-6549 (1992).

54. Mitternacht, S. FreeSASA: an open source C library for solvent accessible surface area calculations. F1000Res. 5, 189 (2016)

55. Hernandez, L. M., Lee, P. D. \& Camacho-Hubner, C. Isolated growth hormone deficiency. Pituitary 10, 351-357 (2007).

56. Alba, M. \& Salvatori, R. Familial growth hormone deficiency and mutations in the GHRH receptor gene. Vitam. Horm. 69, 209-220 (2004).

\section{Acknowledgements}

We thank Chenyao Li for technical assistance. The cryo-EM data were collected at the Center of Cryo-Electron Microscopy, Zhejiang University. This work was partially supported by National Natural Science Foundation of China 81872915 (M.-W.W.), 81922071 (Yan Zhang), 81773792 (D.Y.), 81973373 (D.Y.), 21704064 (Q.Z.), 31770796 (Y.J.), and 31971178 (S.Z.); National Science and Technology Major Project of ChinaKey New Drug Creation and Manufacturing Program 2018ZX09735-001 (M.-W.W.), 2018ZX09711002-002-005 (D.Y.), and 2018ZX09711002-002-002 (Y.J.); Shanghai Municipal Science and Technology Major Project 2019SHZDZX02 (H.E.X.); Ministry of Science and Technology of China Major Project XDB08020303 (H.E.X.); National Key R\&D Program of China 2018YFA0507000 (M.-W.W. and S.Z.); Zhejiang Province Science Fund for Distinguished Young Scholars LR19H310001 (Yan Zhang); Shanghai Science and Technology Development Fund 18ZR1447800 (L.-H.Z.); Young Innovator Association of CAS (2018325 to L.-H.Z.); SA-SIBS Scholarship Program (L.-H.Z.), and Novo Nordisk-CAS Research Fund grant NNCAS-2017-1-CC (D.Y.).

\section{Author contributions}

F.Z., Z.C., and L.-H.Z. designed the expression constructs, purified the GHRH-GHRHR-G $\mathrm{G}_{\mathrm{s}}$ complex, prepared the final samples for negative-stain/data collection toward the structure, and participated in manuscript preparation; H.Z. and C.Y.M. performed specimen screening, cryo-EM grid preparation/data collection, map calculation, and the bulk figures preparation; Q.Z. performed structural analysis, MD simulations on GHRHR, mutation studies, and manuscript preparation; X.C. conducted MD simulations on GHRHR and participated in manuscript preparation; D.-D.S. and C.M. assisted in the specimen screening by negative-stain EM; X.Q.C., Y.W., A.D., and Yan Zhou performed ligand binding and signaling experiments; W.S. and F.H.Z. helped construct preparation, protein expression, and purification; S.Z. and H.J. supervised MD simulations; Y.J. participated in data analysis and manuscript editing; D.Y. supervised ligand binding and signaling experiments, participated in data analysis, and manuscript preparation; H.E.X., Yan Zhang, and M.-W.W. initiated the project, supervised the studies, analyzed the data, and wrote the manuscript with inputs from all co-authors.

\section{Competing interests}

The authors declare no competing interests. 


\section{Additional information}

Supplementary information is available for this paper at https://doi.org/10.1038/s41467020-18945-0.

Correspondence and requests for materials should be addressed to D.Y., H.E.X., Y.Z. or M.-W.W.

Peer review information Nature Communications thanks Javier Garcia-Nafria, Asuka Inoue, and Zhi-Jie Liu for their contribtions to the peer review fof this work.

Reprints and permission information is available at http://www.nature.com/reprints

Publisher's note Springer Nature remains neutral with regard to jurisdictional claims in published maps and institutional affiliations. (c) (i) Open Access This article is licensed under a Creative Commons Attribution 4.0 International License, which permits use, sharing, adaptation, distribution and reproduction in any medium or format, as long as you give appropriate credit to the original author(s) and the source, provide a link to the Creative Commons license, and indicate if changes were made. The images or other third party material in this article are included in the article's Creative Commons license, unless indicated otherwise in a credit line to the material. If material is not included in the article's Creative Commons license and your intended use is not permitted by statutory regulation or exceeds the permitted use, you will need to obtain permission directly from the copyright holder. To view a copy of this license, visit http://creativecommons.org/ licenses/by/4.0/.

(C) The Author(s) 2020 